\title{
FACE RECOGNITION ALGORITHM BASED ON ORIENTATION HISTOGRAM OF HOUGH PEAKS
}

\author{
Amrita Biswas ${ }^{1}$ and M.K.Ghose ${ }^{2}$ \\ ${ }^{1}$ Department of Electronics \& Communication Engineering, SMIT, Majitar, Rangpo \\ ${ }^{2}$ Dean Academics, SMIT, Majitar, Rangpo
}

\begin{abstract}
In this paper we propose a novel face recognition algorithm based on orientation histogram of Hough Transform Peaks. The novelty of the approach lies in utilizing Hough Transform peaks for determining the orientation angles and computing the histogram from it. For extraction of feature vectors first the images are divided into non overlapping blocks of equal size. Then for each of the blocks the orientation histograms are computed. The obtained histograms are combined to form the final feature vector set. Classification is done using $k$ nearest neighbor classifier. The algorithm has been tested on the ORL database, Yale B Database \& the Essex Grimace Database.97\% Recognition rates have been obtained for ORL database, 100\% for Yale B and 100\% for Essex Grimace database
\end{abstract}

\section{KEYWORDS}

Face Recognition, Hough Transform Peaks, Orientation Histograms

\section{INTRODUCTION}

Face Recognition is a challenging field in pattern recognition and computer vision. Several researchers have come forward with various methods for face recognition [1-2]. However recognition under challenging conditions like illumination variation, pose variation, aging etc. still remains an issue. Feature extraction is an essential part of face recognition. Usually a face image contains lot of redundant information which are not essential for recognition purposes. Only specific features like eyes, structure of nose, lips, hair line are more than enough to identify a face image. Also the features extracted should be robust, inavariant to illumination \& pose variations, easy to compute and compact so that storage space required is minimal. Transform based feature extraction processes aim to decorrelate the pixels and pack as much information as possible into the smallest number of transform coefficients[3].Various transform based recognition algorithms have been proposed by researchers[4-7].Transformations like Fourier Transform, Discrete Wavelet Transform, Radon Transform, Fourier Mellin Tranform etc. have been investigated for face recognition. Hough Transform has been widely used by researchers for determining lines and other shapes in pattern recognition problems. Several researchers have utilized Hough Transform for face detection. Arindam Kar et al [8] used Hough Transform for investigating its performance in face recognition. However in their work they computed the Hough Transform of the binary gradient image and in their work selection of significant blocks requires several iterations. In this paper we investigate the Hough Transform based histogram DOI : 10.5121/ijaia.2014.5509 
features for face recognition. Invariance to image scaling, rotation and partial invariance to illumination is an essential feature of Hough Transform [9]. The results obtained using orientation histograms of Hough Transform are as good as the results obtained using state of the art methods used for face recognition.

In the proposed algorithm first the images are divided into non overlapping blocks. Then each block is binarized and the Hough Transform of each binarized block is computed. Finally the Hough Peaks have been detected.30 Hough transform peaks were selected from each image block and then the orientation angle $\theta$ corresponding to the Hough peaks were selected. The orientation histogram for the selected $\theta$ peaks was computed. The orientation bins range from $-90^{\circ}$ to $90^{\circ}$.The same procedure is repeated for each of the blocks and to form the final feature vector set the orientation histograms obtained from each image block are concatenated.

The next sections are divided as follows. In Section 2 we describe the Hough Transform and in Section 3 we describe the concept of Orientation Histograms. In Section 4 the experiments performed and results obtained have been discussed and we finally conclude in section 5 .

\section{HOUGH TRANSFORM}

Hough Transform is a feature extraction technique widely used in image analysis, computer vision and digital image processing. Hough Transform performs groupings of edge points into object candidates by performing an explicit voting procedure over a set of parameterized image objects and thus helps in selecting the edge features of the face images which play an important role in face recognition [10]. The standard Hough transform which is used for detecting straight lines has been used in the proposed algorithm. In the image space, considering a point $\left(x_{i}, y_{i}\right)$ in the $x y$ plane and the general equation of a straight line in slope intercept form $y_{i}=a x_{i}+b$. Plenty of lines pass through $\left(x_{i}, y_{i}\right)$ but they all satisfy the equation $y_{i}=a x_{i}+b$ for varying values of $a$ and $b$. Let us write this equation as $b=-x_{i} a+y_{i}$ and consider the $a b$ plane yields the equation of a single line for a fixed pair $\left(x_{i}, y_{i}\right)$.A second point $\left(x_{j}, y_{j}\right)$ also has a line in parameter space associated with it and unless and until they are parallel this line intersects the line associated with $\left(x_{i}, y_{i}\right)$ at some point $\left(a^{\prime}, b^{\prime}\right)$ where $a^{\prime}$ is the slope and $b^{\prime}$ is the intercept of the line containing both $\left(x_{i}, y_{i}\right)$ and $\left(x_{j}, y_{j}\right)$ in the $x y$ plane. Precisely, all the points on this line have lines in parameter space that intersect at $\left(a^{\prime}, b^{\prime}\right)$.Fig. 1 shows this concept. In principle the parameter space lines corresponding to all points $\left(x_{k}, y_{k}\right)$ in the $x y$ plane could be plotted and the principal lines in that plane could be found by identifying points in parameter space where large numbers of parameter space lines intersect. However, vertical lines give rise to unbounded values of the parameters $a$ and $b$.i.e slope of the line will approach infinity as the line approaches vertical direction. So the other option is to use the normal representation of the line: $x \cos \theta+y \sin \theta=r$

Fig.2(a) illustrates the geometrical interpretation of the parameters $r \& \theta$. A horizontal line has $\theta=0^{\circ}$, with $r$ being equal to the positive $\mathrm{x}$ intercept. Similarly a vertical line has $\theta=90^{\circ}$, with $r$ being equal to the positive y intercept or $\theta=-90^{\circ}$, with $r$ being equal to the negative y intercept. Each sinusoidal curve in Fig.2(b) represents the family of lines that pass through a particular point $\left(x_{k}, y_{k}\right)$ in the xy plane. The intersection point $\left(r^{\prime}, \theta^{\prime}\right)$ in Fig.2(b) corresponds to the line that passes through both $\left(x_{i}, y_{i}\right)$ and $\left(x_{j}, y_{j}\right)$ in Fig.2(a). The $r \theta$ parameter space is subdivided into accumulator cells shown in Fig.2(c) where $\left(r_{\min }, r_{\max }\right)$ and $\left(\theta_{\min }, \theta_{\max }\right)$ are the expected ranges of the parameter values : $-90^{\circ} \leq \theta \leq 90^{\circ}$ and $-D \leq r \leq D$, where $D$ is the maximum distance between opposite corners of the image. The cell at coordinates $(i, j)$ with accumulator values $A(i, j)$ 
,corresponds to the square associated with parameter space coordinates $\left(r_{i}, \theta j\right)$.Initially these cells are set to zero. Then for every non background pixel $\left(x_{k}, y_{k}\right)$ in the $x y$ plane $\theta$ is equaled each of the allowed subdivision values on the $\theta$ axis and solved for the corresponding value of $r$ using the equation : $r=x_{k} \cos \theta+y_{k} \sin \theta$

The resulting $r$ values are then rounded off to the nearest allowed cell value along the $r$ axis. If a choice of $\theta_{p}$ results in solution $r_{q}$, then $A(p, q)=A(p, q)+1$. After the procedure is over a value of $P$ in $A(i, j)$ means that $P$ points in the $x y$ plane lie on the line $x \cos \theta_{j}+y \sin \theta_{j}=r_{i}$. The number of computations in this method is linear with respect to the number of non background points in the $x y$ plane.

An approach based on Hough Transform is as follows:

- Obtain binary edge image

- Specify subdivisions in the $r \theta$ plane.

- Examine the counts of the accumulator cells for high pixel concentrations.

- Examine the relationship (principally for continuity) between pixels in a chosen cell.
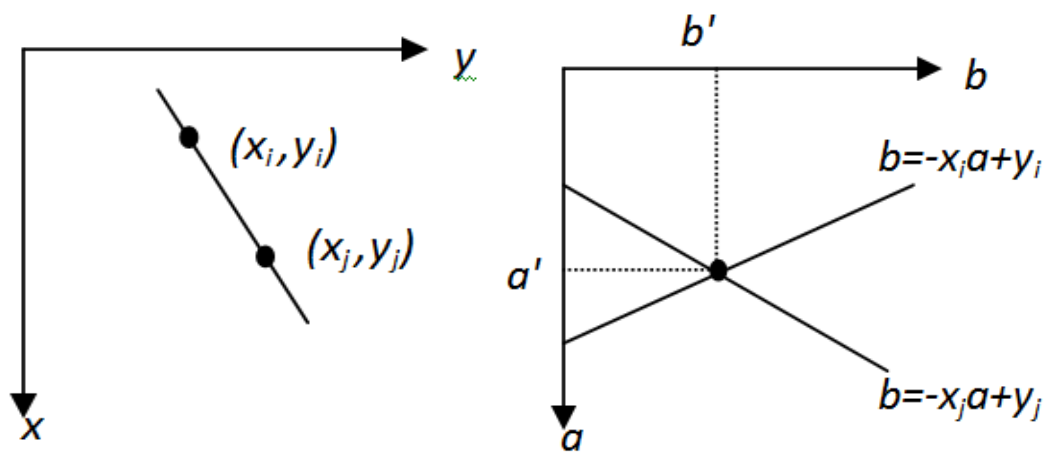

(a)

Fig. 1 (a) xy plane (b) Parameter Space

(b)

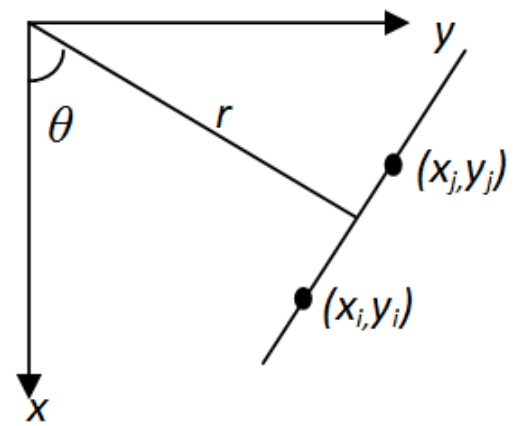

(a) 


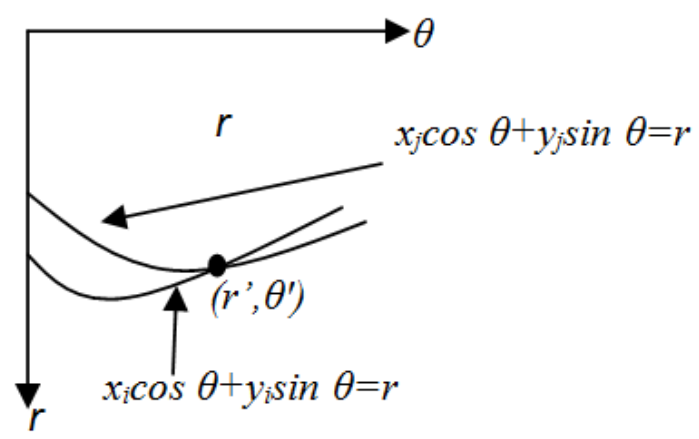

(b)

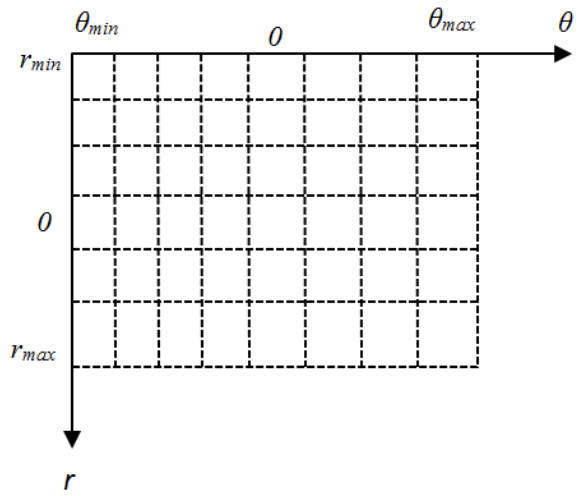

(c)

Fig.2. $(a)(r, \theta)$ parameterization of line in xy plane (b) Sinusoidal curves in the $(r, \theta)$ plane; the point of intersection $\left(\mathrm{r}^{\prime}, \theta^{\prime}\right)$ corresponds to the line passing through points $\left(\mathrm{x}_{\mathrm{i}}, \mathrm{y}_{\mathrm{i}}\right)$ and $\left(\mathrm{x}_{\mathrm{j}}, \mathrm{y}_{\mathrm{j}}\right)$ in the xy plane (c) Division of the $(r, \theta)$ plane into accumulator cells

\section{ORIENTATION HISTOGRAMS}

The histogram of a digital image with gray levels in the range [0, L-1] is a discrete function $h\left(r_{k}\right)$ $=n_{k}$, where $r_{k}$ is the $k$ th gray level and $n_{k}$ is the number of pixel in the image having gray level $r_{k}$. It is common practice to normalize a histogram by dividing each of its values by the total number of pixels in the image, denoted by $n$. Thus, a normalized histogram is given by $p\left(r_{k}\right)=n_{k} / n$, for $k$ $=0,1 \ldots \ldots, L-1$. In other words, $p\left(r_{k}\right)$ gives an estimate of probability of occurrence of gray level $r_{k}$. The motivation behind the Orientation Histogram descriptors is that local object appearance and shape within an image can be described by the distribution of intensity gradients or edge directions. These descriptors can be computed by dividing the image into small blocks, and for each block computing a histogram of the orientation $\theta$.The concatenation of these histograms will form the descriptor. To improve the efficiency, the local histograms can be normalized by calculating a measure of the intensity across a larger region of the image, and then using this value to normalize all blocks within that region. Invariance to changes in illumination or shadowing improves due to this normalization. The major advantages of this descriptor will be invariance to geometric and photometric transformations and hence the feature vectors obtained from these descriptors are well suited for application in face recognition problems.

\section{PROPOSED APPROACH}

- The images are partitioned into equal sized non overlapping blocks

- The gray level block are converted into binary form

- The Hough Transform of each image block is computed.

- The Hough Peaks are detected and the first 30 Hough peaks are retained

- The orientation angle $\theta$ for the retained peaks are selected

- Based on $\theta$ the orientation histograms are computed for the image blocks

- The histograms of all the blocks were concatenated to form the final feature vector set 
International Journal of Artificial Intelligence \& Applications (IJAIA), Vol. 5, No. 5, September 2014

\section{EXPERIMENTS PERFORMED \& RESULTS OBTAINED}

For our experiments we have tested the ORL, Yale B \& Essex Grimace Database. The Databases are described as follows:

ORL (AT and T) database has 10 different images (92 x 112), each of 40 different subjects. All images were taken against a dark homogenous background with the subjects in upright, frontal position with some side movement. Sample images of the ORL database have been shown in Fig. 2. Yale Database contains images of 10 subjects (480x 640) under 64 different lighting conditions. Sample images of this database are shown in Fig. 3. Essex-Grimace database has a set of 20 different images (180x200), each of 18 individuals. The database contains images of both male and female subjects, taken with a fixed camera. Sample images of the Essex Grimace database have been shown in Fig. 4.

All the images from the three databases were resized to $112 \times 112$.Then the images were divided into blocks of size 16x16.So a total of 49 blocks were obtained for each image. Each of the gray level blocks was converted to binary and the Hough Transform for each image block was computed. The size of the obtained Hough Transformed matrix for each image block of size $16 \times 16$ is $45 \times 1796$.Next the Hough Peaks were detected and a total of 30 peaks were retained for each image block. The orientation angle $\theta$ for the 30 peaks were selected and the orientation histogram computed for the image blocks. The orientation bins range from $-90^{\circ}$ to $90^{\circ}$. Hence the size of the histogram obtained for each block is $1 \mathrm{x} 181$. The orientation histograms thus obtained were concatenated to form the final feature vector set of the given image. Hence the size of the feature vector set for a given image is $1 \times 8869$

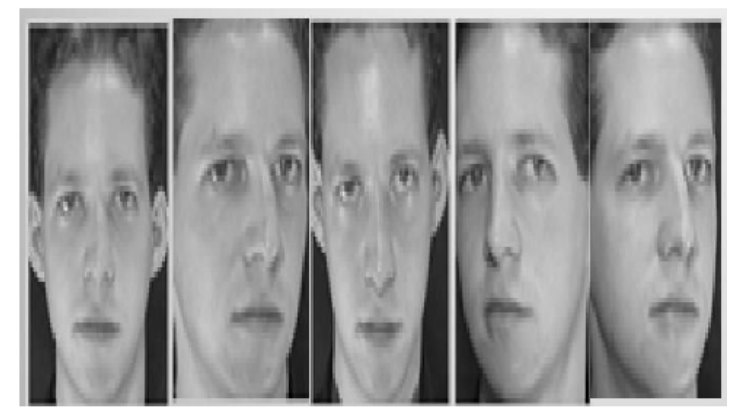

Fig.2.Sample Images of the ORL Database

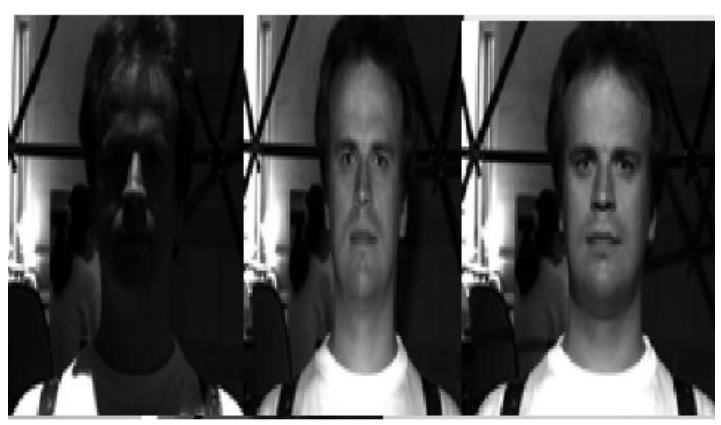

Fig.3.Sample Images of the Yale B Database 
International Journal of Artificial Intelligence \& Applications (IJAIA), Vol. 5, No. 5, September 2014

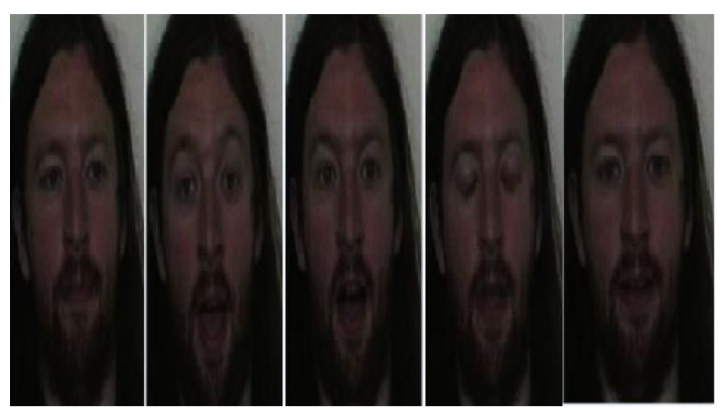

Fig.4.Sample Images of the Essex Grimace Database

For each of the databases we have taken different numbers of training images and the remaining images were selected for testing. k nearest neighbor classifier was used for classification.
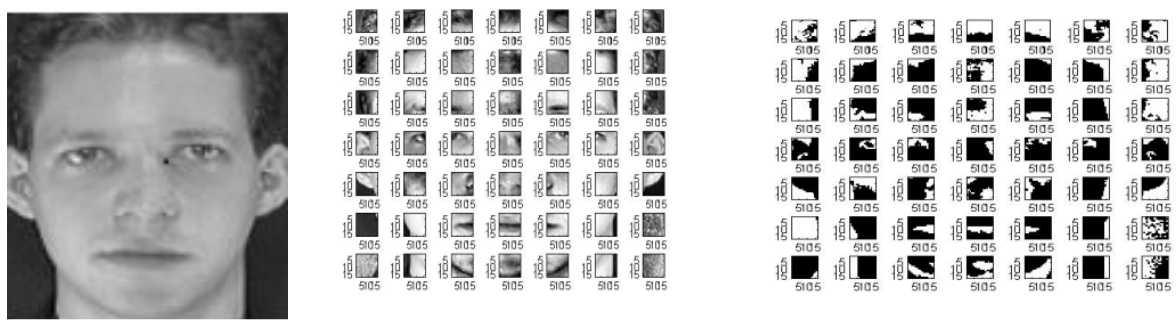

Fig.5(a)

Fig.5(b)

Fig.5(c)

Fig.5(a) Original Image (b) Image Partitioned into blocks of size 16x16 (c) Binarized blocks of Image

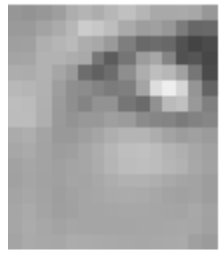

Fig.6(a)

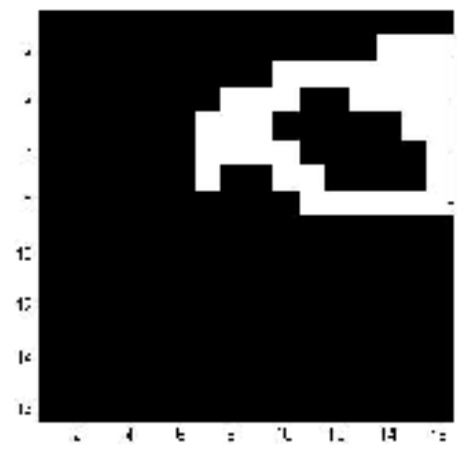

Fig.6(b) 
International Journal of Artificial Intelligence \& Applications (IJAIA), Vol. 5, No. 5, September 2014

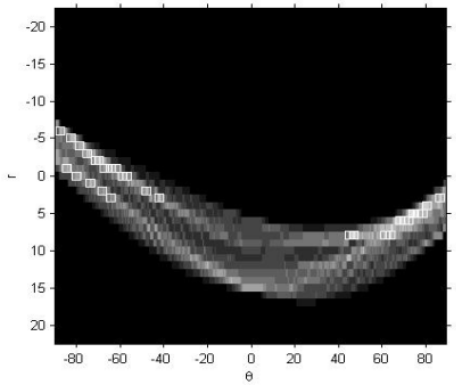

Fig.6(c)

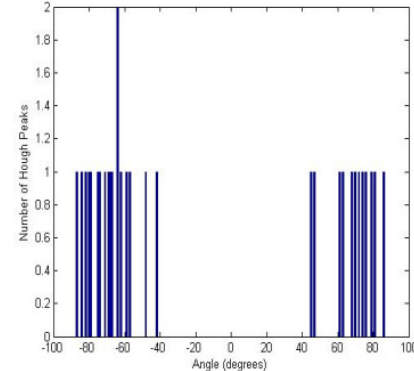

Fig.6(d)

Fig.6(a) One of the image blocks of size 16x16 (b) Corresponding binarized image block (c) Hough Transform image \& the 30 Peaks extracted from the same block (d) Orientation Histogram of Hough Peaks for the same block

Results obtained have been shown in Table.1.

Table 1: Results obtained

\begin{tabular}{|l|l|c|c|}
\hline Sl.No. & Database & $\begin{array}{c}\text { No.of } \\
\text { Training } \\
\text { Images }\end{array}$ & Recognition \% \\
\hline 1 & ORL & 5 & 97 \\
\hline 2 & ORL & 4 & 96 \\
\hline 3 & ORL & 3 & 92 \\
\hline 4 & ORL & 2 & 90 \\
\hline 5 & ORL & 1 & 80 \\
\hline 6 & Yale B & 4 & 100 \\
\hline 7 & Yale B & 3 & 97 \\
\hline 8 & Yale B & 2 & 96 \\
\hline 9 & Yale B & 1 & 94 \\
\hline 10 & Essex- & 1 & 100 \\
\hline & Grimace & & \\
\hline
\end{tabular}

Table 2.Comparison with other transform based algorithms for single training image

\begin{tabular}{|c|c|c|c|}
\hline Sl.No & Algorithm & Database & Recog\% \\
\hline 1 & DWT based Algorithm using coiflets[4] & ORL & 71.2 \\
\cline { 3 - 4 } & & Yale & 67.5 \\
\cline { 3 - 4 } & & Essex Grimace & 85.3 \\
\hline 2 & Radon Transform based Algorithm[4] & ORL & 67.3 \\
\cline { 3 - 4 } & & Yale & 70 \\
\cline { 3 - 4 } & & Essex Grimace & 97 \\
\hline 3 & \multirow{2}{*}{ FMT based Algorithm[5] } & ORL & 68 \\
\cline { 3 - 4 } & & Yale & 65 \\
\hline 4 & \multirow{2}{*}{ Proposed Orientation Histogram based Algorithm } & ORL & $\mathbf{8 0}$ \\
\cline { 3 - 4 } & & Yale & $\mathbf{9 4}$ \\
\cline { 3 - 4 } & & & $\mathbf{1 0 0}$ \\
\hline
\end{tabular}


International Journal of Artificial Intelligence \& Applications (IJAIA), Vol. 5, No. 5, September 2014

\section{CONCLUSION}

From the results obtained it can be seen that the orientation histograms obtained from the peaks of the Hough Transformation matrix does a good job in minimizing the effects of illumination variance. The algorithm performs well on the Yale B database which consists of images with major illumination variance. It also takes care of major expression variations which can be corroborated by the results obtained on the Essex Grimace Database. The orientation histograms also take care of the pose variations in the ORL database and it can be seen that as we increase the number of images in the training database the performance of the algorithm improves. The best performance of the proposed approach for the three databases is almost similar to the transform based algorithms proposed in [4] and [5].But it can be said that when the number of training images reduce there is significant reduction in performance of the transformed based algorithms but the Orientation Histogram based algorithm performs better for less number of training images. One of the reasons could be the orientation histograms better depict the rotation variance of the images. Also, while the transform based approaches were holistic, the Orientation Histogram based approach is local feature based. The histograms contain information about the distribution of the local orientation over the whole image. But for efficient face representation spatial information also has to be retained. To retain spatial information the image is has been partitioned into non overlapping blocks and combination of the histograms of these blocks provide the spatially enhanced histogram. The results reflected in the comparison table provide inspiration to investigate local appearance based methods for designing robust algorithms using single training image.

\section{REFERENCES}

[1] Rabia Jafri and Hamid R. Arabnia,2009,A survey of Face Recognition Techniques, Journal of Information Processing Systems, Vol.5,No.2,pp-41-68

[2] Ripal Patel, Nidhi Rathod, Ami Shah, 2012,Comparitive Analysis of Face Recognition Approaches:A Survey, Vol:57,No.17,pp-50-69

[3] R. C. Gonzalez and R. E. woods," Digital Image Processing”, Dorling Kindersley, India, Pearson Prentice Hall, 2006.

[4] Sambhunath Biswas,Amrita Biswas,2012,Face Recognition Algorithms Based on Transformed Shape Features, IJCSI International Journal of Computer Science Issues, Vol. 9, Issue 3, No 3,pp-445-451

[5] Sambhunath Biswas and Amrita Biswas,2013,FourierMellin Transform Based Face Recognition,International Journal of Computer Engineering and Technology(IJCET),vol.4,issue 1, pp-8-15

[6] Anissa Bouzalmat, Arsalane Zarghili and Jamal Kharroubi,2011, Facial Face Recognition Method using Fourier Transform Filters Gabor and R_LDA, IJCA Special Issue on Intelligent Systems and Data Processing, pp-18-24.

[7] Zheng Dezhong Cui Fayi, 'Face Recognition based on Wavelet Transform and Image Comparison",2008,Proc. International Symposium on Computational Intelligence and Design, Volume: 2, pp-24-29.

[8] Arindam Kar1, Debotosh Bhattacharjee2, Dipak Kumar Basu2, Mita Nasipuri2, Mahantapas Kundu2,2012,Face Recognition using Hough Peaks extracted from the significant blocks of the Gradient Image, International Journal of Advanced Research in Computer Science and Software Engineering,Vol.2,Issuue 1,pp-1-6.

[9] http://homepages.inf.ed.ac.uk/rbf/HIPR2/hough.htm.

[10] Shapiro, Linda and Stockman, George. "Computer Vision", Prentice-Hall, Inc. 2001

[11] "Use of the Hough Transformation to Detect Lines and Curves in Pictures" (http:/ / www. ai. sri. com/ pubs/ files/ tn036-duda71. pdf).

[12]R.C Gonzalez and R.E Woods ,Digital Image Processing,Dorling Kinderlsey,India,Pearson,Prentice Hall,2006 